photocoagulation therapy for their AE. On logistic regression, factors associated with a subsequent change in management included the indication of $\mathrm{OB}(\mathrm{p}=0.03)$, the presence of comorbidity $(p=0.03)$, transfusion dependence $(p=0.02)$ and the presence of AE $(p<0.001)$ on the CE. In $14 \%$ of patients, significant lesions were found outside the SB (upper GI tract $12 \%$ and colon $2 \%$ ).

Conclusion This is the largest series on the use of CE in OGB in the published literature. A significant number of patients with a positive DY had a change in management. Factors that were more likely to predict an alteration of outcome were the presence of comorbidity, transfusion dependence and the presence of $\mathrm{AE}$. Although, the DY did not differ significantly between those with IDA and $\mathrm{OB}$, management was more likely to be altered in those with $\mathrm{OB}$

Disclosure of Interest None Declared.

\section{PTH-186 THE UTILITY OF CAPSULE ENDOSCOPY IN PATIENTS WITH IRON DEFICIENCY ANAEMIA UNDER 50 YEARS- IS THE JUICE WORTH THE SQUEEZE?}

doi:10.1136/gutjnl-2013-304907.673

1."P S Sidhu, 'M E McAlindon, 'K Drew, 'R Sidhu. 'Gastroenterology, Royal Hallamshire Hospital, Sheffield Teaching Hospitals, Sheffield, UK

Introduction The role of capsule endoscopy (CE) in patients with iron deficiency anaemia (IDA) under the age of 50 years remains unclear. There is paucity of data on its use in this age group. The aim of this study was to assess the utility of CE in patients under the age of 50 years in comparison to the older cohort.

Methods All patients referred for CE routinely for IDA were included. Patients were divided into group 1 ( $<50$ years) and group 2 ( $\geq 50$ years). Data was collected retrospectively for demographics, the presence of co-morbidity, medications including anticoagulation, diagnostic yield and subsequent follow up. The data was analysed using SPSS Ver 18.

Results There were 971 patients with IDA identified with $28 \%$ of patients belonging to group 1 . The mean age was 40 years in this group (range $17-49$ years) with $61 \%(n=168)$ of the cohort being female. The diagnostic yield in group 1 was $28 \%(n=76)$ with no difference between the sexes. Significant diagnoses were found in this age group including erosions and ulcers $26 \%(n=71)$, small bowel (SB) angioectasia (AE) in 10\% $(n=27)$, SB tumours (7), crohn's disease (7), SB bowel strictures (3), and SB varices (2). Other findings (14) included endometriosis, a dielaufoy lesion, diverticulum and fresh blood from uncertain site. On logistic regression, the presence of diabetes $(p=0.02)$ and the use of warfarin $(p=0.049)$ was associated with increased yield. Management was altered in $59 \%(n=57)$ of patients with a positive yield on CE. This was in the form of double balloon enteroscopy (11), push enteroscopy (2), repeat OGD (2), surgery (2), repeat CE (5) with only one patient receiving argon photocoagulation therapy in this group. On logistic regression, clinical factors that were associated with a management change included the presence of rheumatology disease $(p=0.006)$, diabetes $(p=0.014)$, previous transfusion $(p=0.01)$ and SB AE $(p=0.002)$ in group 1 . The diagnostic yield in group 2 was $38 \%$ which was significantly higher than in group $1(p=0.02)$. Whilst small bowel tumours were equally common in both groups, angioectasia was commoner in group $2(\mathrm{p}<0.001)$.

Conclusion A significant proportion of patients below 50 years are referred for CE. Although the diagnostic yield is lower compared to those above 50 years, significant pathology is found in this age group. CE should be considered in the management pathway of patients under 50 years with recurrent IDA in both sexes.

Disclosure of Interest None Declared.

\section{PTH-187 A NOVEL NON-INVASIVE APPROACH TO DIAGNOSE BILE ACID DIARRHOEA (BAD) USING AN ELECTRONIC NOSE (E-NOSE) AND FIELD ASYMIMETRIC ION MOBILITY SPECTROSCOPY (FAIMS)}

doi:10.1136/gutjnl-2013-304907.674

1J Covington, ${ }^{2} \mathrm{E}$ Westinbrink, ${ }^{2} \mathrm{M}$ Thomas, ${ }^{3} \mathrm{~N}$ O'Connell, ${ }^{3} \mathrm{C}$ Bailey, ${ }^{3} \mathrm{C}$ Nwokolo, ${ }^{4} \mathrm{~J}$ Cullis, ${ }^{4} \mathrm{~N}$ Williams, ${ }^{5} \mathrm{~K}$ Bardhan, ${ }^{6,} \mathrm{R}$ Arasaradnam. ${ }^{1}$ School of Engineering; ${ }^{2}$ University of Warwick: ${ }^{3}$ University Hospital Coventry \& Warwickshire, Coventry, UK; ${ }^{4}$ Nuclear Medicine, University Hospital Coventry \& Warwickshire, Coventry; ${ }^{5}$ Rotherham NHS Foundation Trust, Rotherham; ${ }^{6}$ Clinical Sciences Research Institute, University of Warwick, Coventry, UK

Introduction A third of diarrhoea predominant irritable bowel syndrome (D-IBS) results from bile acid malabsorption (BAM); diagnosed by ${ }^{75} \mathrm{SeHCAT}$ retention test. We have previously demonstrated the ability of using an E-nose and FAIMS to detect inflammatory bowel disease (IBD) by shifts in the patterns of volatile organic compounds (VOCs) in the gases and vapours that emanate from urine samples. Here, we have extended our work to detect BAM from urine odours alone.

Methods Technology Principles - E-nose This uses an array of gas phase chemical sensors which are broadly tuned to different chemical groups. When a urine sample is presented to the sensor array, a unique response is produced. By taking all of the sensor responses together, a bio-odorant fingerprint is created. FAIMS operates on similar principles, but produces its fingerprint by measuring the differences in mobility of ionised chemicals in high electric fields.

Subjects and patients BAM (7 day retention value of $<15 \%$ ): $\mathrm{n}=15$. Controls: ulcerative colitis in remission (SCAI score of $<4$ ): $\mathrm{n}=20$; healthy subjects: $\mathrm{n}=7$. Urine was collected in $10 \mathrm{ml}$ aliquots and stored frozen in universal containers. For assay, the containers were first heated to $60 \pm 0.1^{\circ} \mathrm{C}$. The headspace (the air above the sample) was then pumped from the containers and analysed by an AlphaMOS FOX 4000 E-nose and FAIMS instrument. Discriminant Function Analysis and Fisher Discriminant Analysis (FDA) were used for statistical evaluation, respectively.

Results The FOX 4000 E-nose plot (Figure 1) shows separate and distinct groupings of patients with BAM, UC, and healthy controls. FAIMS analysis gave a broadly similar pattern (not shown).

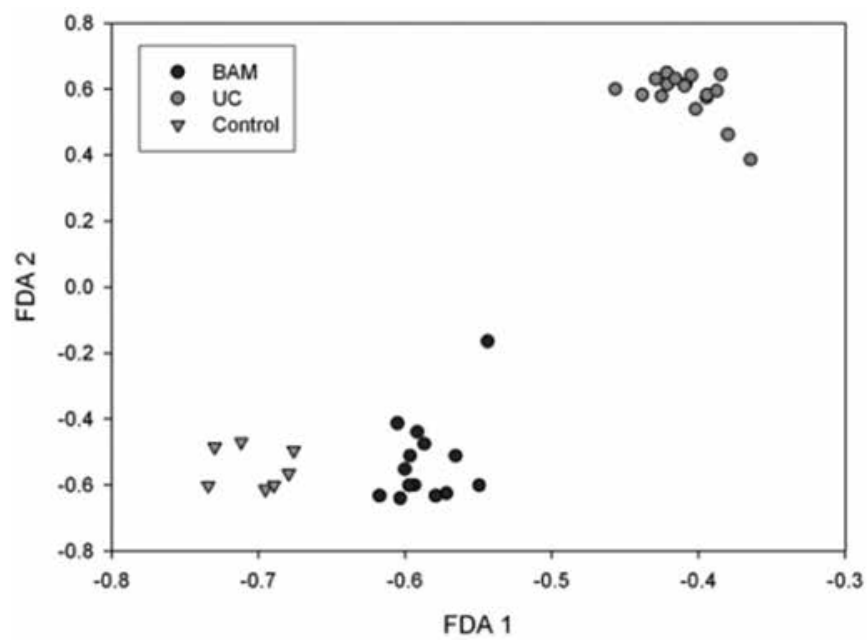

\section{Abstract PTH-187 Figure 1}

Conclusion D-IBS related to BAM is a common condition and treatment with BA sequestrants is effective in the majority. The SeHCAT retention test makes diagnosis easy but is not available in all centres: our pilot data offers an alternative diagnostic approach with high potential.

Disclosure of Interest None Declared. 